7-1-1981

\title{
Spatial Aggregation in Multiregional Input-Output Models
}

Peter Blair

Ronald E. Miller

Follow this and additional works at: https://researchrepository.wvu.edu/rri_iotheorymethods

\section{Recommended Citation}

Blair, Peter and Miller, Ronald E., "Spatial Aggregation in Multiregional Input-Output Models" (1981).

Theory and Methods. 48.

https://researchrepository.wvu.edu/rri_iotheorymethods/48

This Article is brought to you for free and open access by the RRI Input-Output Archive at The Research Repository @ WVU. It has been accepted for inclusion in Theory and Methods by an authorized administrator of The Research Repository@WVU. For more information, please contact beau.smith@mail.wvu.edu. 
Submitted to Environment and Planning A, October 1981

SPATIAL AGGREGATION IN

MULTIREGIONAL INPUT-OUTPUT MODELS

Peter Blair*

Ronald E. Miller**

Working Paper No. 52

REGIONAL RESEARCH INSTITUTE

WORKING PAPERS IN

REGIONAL SCIENCE AND TRANSPORTATION

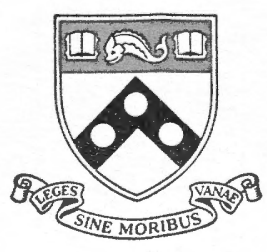

UNIVERSITY of PENNSYLVANIA

PHILADELPHIA, PENNSYLVANIA 19104 
SPATIAL AGGREGATION IN

MULTIREGIONAL INPUT-OUTPUT MODELS

\author{
Peter Blair* \\ Ronald E. Miller** \\ Working Paper No. 52
}

July, 1981

This work has been supported by NSF grant SOC-7907215 which is gratefully acknowledged.

*Assistant Professor of Regional Science and Public Policy **Professor of Regional Science 
Abstract

Page

1 INTRODUCTION

2 INTERREGIONAL AND MULIIREGIONAL INPUT-OUTPUT MODELS

2

3 INITIAL EXPERIMENTS: RANDOM MATRICES 6

3.1 Generating Multiregional from Interregional Data 6

3.2 General Structure of the Random Matrix Experiments 8

3.3 Strength of Spatial Linkages 11

3.4 Meausres of Error 12

4 RESLLTS FROM THE RANDOM MATRIX EXPERIMENTS 14

5 RESULTS FROM EXPERIMENTS WITH THE U.S. MUTIREGIONAL DATA 22

6 CONCLLSIONS 26

Appendix A: Generating Multiregional from Interregional Data -- An $\begin{array}{ll}\text { Illustration } & 27\end{array}$

Appendix B: Regions and Sectors in the U.S. Multiregional Data 32

References $\quad 34$ 
This study continues the investigations into effects of spatial aggregation that were initially reported upon in Miller and Blair (1980) for an interregional input-output model. In the present paper, our interest is in the error that is introduced into a multiregional input-ouput model through aggregation of regions. As in the earlier paper, we investigate the question using both hypothetical and real data. In the present study, these are (1) randomly-generated multiregional data incorporating varying degrees of regional linkage and (2) actual 1963 U.S. multiregional data. To allow some comparison with the interregional experiments, the data for the random experiments are derived from our original randomly-generated interregional data. The same levels of spatial interconnectedness as in the interregional experiments are also used here, and the same measures of aggregation error are employed. As in the interregional case, we conclude that spatial aggregation in multiregional input-output models does not necessarily lead to unacceptable error. 
SPATIAI AGGREGATION IN MULTIREGIONAL INPUT-OUTPUT MODELS

\section{INTRODUCTION}

This paper reports the results of a set of experiments designed to examine the effects of spatial, as opposed to sectoral, aggregation in multiregional input-output models. It is entirely similar in objectives to our earlier report on the effects of such aggregation in interregional input-output models [Miller and Blair (1980)]. One principal conclusion of this earlier paper was that, by our measures, the amount of error introduced by spatial aggregation into the results of analyses using the interregional input-output framework was remarkably small in a rather wide variety of cases. Since there is a well-known and quite widely used multiregional input-output model for the United States [Polenske (1980)], it may be of even more practical concern to know how important spatial detail is in the multiregional framework. The experiments are very similar in structure to those in our earlier paper, and readers are referred to that paper for details. In the present paper, we recall the structure of the multiregional inputoutput model in section 2. Then, in Section 3, we describe the initial experiments, using data from the earlier interregional cases. Sections 4 and 5 contain results from the experiments using the random data and the actual U.S. multiregional data, respectively. In section 6 we offer some concluding thoughts. Appendix A contains a numerical example to illustrate how the random data originally generated for the interregional model are converted for use in the multiregional case. Appendix B contains the regional and sectoral breakdowns for the nine-region, ten-sector multiregional input-output model used in Section 5. 
In the interregional input-output model, it is necessary to have data on sales from sector $i$ in region $s$ to sector $j$ in region $T, z_{i j}^{S T}$, for all sectors and all regions (including intraregional sales, when $S=T$ ).

For a model with three sectors in each of two regions, $I$ and $M$, the $(6 \times 6)$ matrix

$$
z=\left\{\begin{array}{l:l}
z^{L I} z^{L M} \\
\hdashline z^{M L} & z^{M M}
\end{array}\right\}
$$

where $z^{S T}=\left[\begin{array}{c}S T \\ i j\end{array}\right]$, represents all interindustry flows, both intraregional -the two $(3 \times 3)$ on-diagonal blocks -- and interregional -- the two $(3 \times 3)$ off-diagonal blocks. Given sales to final demand in each region, $Y^{I}$ and $Y^{M}$ (each a three-element vector), and forming

$$
Y=\left\{\frac{Y^{L}}{Y^{M}}\right\}
$$

then the vectors of total outputs of each sector in each region, $x^{L}$ and $x^{M}$, are found as

$$
X=-\frac{X^{L}}{X^{-}}=2 i+Y
$$

where $i$ is an appropriately-sized column vector containing ones. The matrix of intraregional and interregional (trade) coefficients

$$
\bar{A}=\frac{A}{L L} A_{A}^{L M}
$$

is found from

$$
\bar{A}=Z(\hat{X})^{-1}
$$


where $\hat{X}$ represents the diagonal matrix formed from the vector $X$. Thus the fundamental input-output relationships are representable as

(4) $(I-\bar{A}) X=Y$

and

$$
X=(I-\bar{A})^{-1} Y
$$

as usual. Thus outputs of sectors in both regions are related to final demands for those sectors.

Explicitly, for this two-region case, the relationships in (4) are

$$
\begin{aligned}
& \left(I-A^{L L}\right) X^{L}-A^{L M} X^{M}=Y^{L} \\
& -A^{M L} X^{L}+\left(I-A^{M M}\right) X^{M}=Y^{M}
\end{aligned}
$$

The multiregional input-output framework represents an attempt to capture these intra- and interregional connections in a model that requires less detailed data than the flows in the matrix $z$ in (1). Specifically, it is assumed that the best estimates of regional technical coefficient matrices are not of the $A^{L L}$ and $A^{M L}$ sort -- inputs of region $L$ goods or of region $M$ goods per dollar's worth of output of region L sectors -- but rather are simply of the $A^{L}$ and $A^{M}$ sort -- technical coefficients for productive activities in region $L$ (or $M$ ), without considering whether or not a specific input is supplied from within or outside of the region. Thus, knowing only $z_{i j}^{\cdot T}$ i.e., how much of good $i$ was bought by sector $j$ in region $T$ (and not knowing the region of origin of the good), allows one to develop regional (not interand intraregional) coefficient matrices. That is, $z_{i j} \cdot T / x_{j}^{T}=a_{i j}^{\cdot T}$ and

$$
A^{T}=\left[\begin{array}{l}
a_{i j}^{T} \\
{[}
\end{array}\right]=\left(z^{T}\right)\left(\hat{X}^{T}\right)^{-1}
$$

where

$$
z^{T}=\left[\begin{array}{l}
\cdot T \\
i j
\end{array}\right]
$$


(We drop the inftial "dot" superscript for notational simplicity.)

In addition, instead of observations on $z_{1 j}^{S T}$, the multiregional inputoutput system assumes that one has data on flows from sector $i$ in region $L$ to all purchasing sectors (including final demand) in region $M$-- that is, the sector of destination is suppressed. Data required are values of shipments, by commodity, between and among the regions; call these $q_{i}^{S T}$.

Based on these two observations on the more likely kind of data to be available, the multiregional input-output framework develops estimates of the interregional connections in (6) as follows. Regional supply percentages are estimated after all flow data for good $i$ have been collected in a flow matrix (to continue with the two-region example) $Q(i)$, where

$$
Q(i)=\left[\begin{array}{cc}
q_{i}^{L L} & q_{i .}^{L M} \\
q_{i .} & q_{i .}^{M M}
\end{array}\right]
$$

This simply records shipments of good $i$ between and among regions (whether for interindustry or final demand use). Note that, for example, the first row sum of $Q(i)$ must equal the total output of good $i$ in region $L$, namely $x_{i}$. Column sums indicate the total supply of commodity $i$ in the receiving region, i.e.,

$$
T_{i}^{L}=c_{i}^{L L}+q_{i}^{M L}
$$

Each element in a column, say the first column in (9), divided by that column sum, $T_{i}^{I}$, represents the proportion of the total amount of good $i$ in region $L$ that comes from each region. Thus,

$$
c_{i}^{L L}=q_{i \cdot}^{L L} / T_{i}^{L}
$$

and 


$$
c_{i}^{M L}=q_{i}^{M L} / T_{i}^{L}
$$

where the c's represent these proportions. Multiplied by 100, these are regional supply percentages. Arranging these proportions for all goods for a given pair of regions.in a vector, one has

$$
c^{L M}=\left[\begin{array}{c}
c_{1}^{L M} \\
c_{2}^{L M} \\
c_{3}^{L M}
\end{array}\right]
$$

And, similarly, one can construct $C^{I L}, C^{M L}$ and $C^{M L}$, all three-element vectors in our two-region, three-sector example.

The crucial assumption in the multiregional input-output model is that these (observable) proportions represent average behavior for all purchasers of each good $i$ imported from region $L$ to sectors in region $M$. If $c_{1}^{L M}=0.4$, then all consumers of good $I$ in region $M$ are assumed to purchase 40 percent of their needs of that good from region $L$. If vectors such as $C^{L M}$ are diagonalized as in

$$
C^{L M}=\left[\begin{array}{ccc}
c_{1}^{L M} & 0 & 0 \\
0 & c_{2}^{L M} & 0 \\
0 & 0 & c_{3}^{L M}
\end{array}\right]
$$

then the product,

$$
\hat{C}^{L M} A^{M}
$$

is the multiregional input-output model's estimate of the interregional relationships embodied in $A^{L M}$. Similarly, $\hat{C}^{L L_{A} L}, \hat{C}^{M L}{ }_{A}$ and $\hat{C}^{M M} A^{M}$ are used as proxies for their interregional counterparts $A^{L L}, A^{M L}$ and $A^{M M}$, respectively. The trade proportions represented by vectors like $C^{L M}$ in (13) also relate to final demand purchases in region $M$, since the underlying flow data, in (9), measure both interindustry and final demand sales. Therefore the multiregional input-output equivalent to the twu-region relationships in (6) is 


$$
\begin{aligned}
& \left(I-\hat{C}^{L L} A_{A}^{L}\right) X^{L}-\hat{C}^{L M_{A} M_{X} M}=\hat{C}^{L L_{Y} L}+\hat{C}^{L M_{Y} M} \\
& -\hat{C}^{M L} A^{L} X^{L}+\left(I-\hat{C}^{M M} A^{M}\right) X^{M}=\hat{C}^{M L} Y^{L}+\hat{C}^{M M} Y^{M}
\end{aligned}
$$

In the multiregional model, $\mathrm{Y}^{\mathrm{L}}$ and $\mathrm{Y}^{\mathrm{M}}$ represent demands in region $\mathrm{L}$ or region $M$ for goods, irrespective of their regional origins. For $Y^{L}$, for example, the $\hat{C}^{L L}$ and $\hat{C}^{M L}$ matrices determine how much of the final demand is satisfied from within $L$ and how much is imported from $M$.

Letting

$$
\hat{A}=\left[\begin{array}{l:l}
A^{I} & \emptyset \\
\hdashline 0 & A^{2}
\end{array}\right]
$$

and

$$
\hat{\mathrm{C}}=\left[\begin{array}{c:c}
\hat{C} L L & \hat{L}{ }^{L M} \\
\hdashline \hat{C} & \hat{C} M
\end{array}\right.
$$

the multiregional counterpart to $\bar{A}$ in (2) is

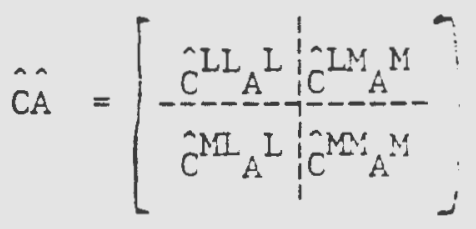

and, with $X=\left[\begin{array}{l}X^{L} \\ X^{M}\end{array}\right]$ and $Y=\left[\begin{array}{c}Y^{I} \\ -\frac{1}{Y}\end{array}\right]$, the counterparts to (4) and (5) are
(20) $\quad(I-\hat{C A}) X=\hat{C Y}$

and

$$
X=(I-\hat{C A})^{-1} \hat{C Y}
$$

\section{INITIAL EXPERIMENTS: RANDOM MATRICES}

\subsection{Generating Multiregional from Interregional Data}

In order to preserve as much comparability as possible with our previous interregional input-output experiments, we have attempted to utilize the uniformly-distributed, randomly-generated flow data that were originally 
produced for those experiments. Thus, for example, instead of generating random flow data $z_{i j}^{T}$, as are needed for the regional coefficient matrices, $A^{T}$, we have derived the $Z^{T}$ data for our current experiments from the earlier, more detailed $\mathrm{z}^{\mathrm{ST}}$ kinds of flows.

The $(50 \times 50) 2$ matrices from our interregional system with five regions of ten sectors each were of the form

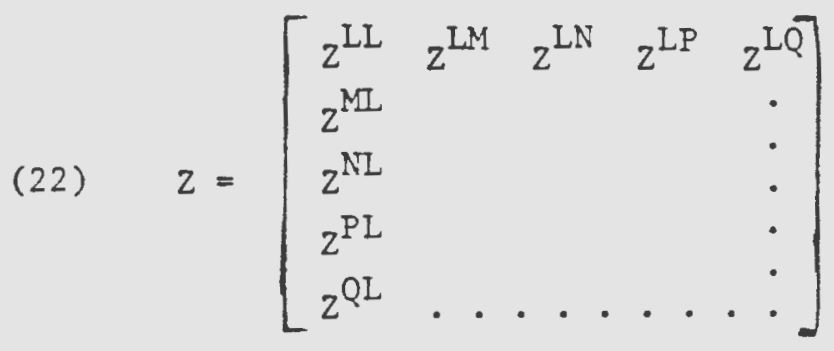

where each submatrix, $z^{S T}$, is $(10 \times 10)$. For the multiregional experiments, we form ( $10 \times 10)$ matrices of the sort $z^{L}$-- as in (8) -- by adding together the matrices in the first (block) column of $z$ in (22). That is,

$$
z^{L}=z^{L L}+z^{M L}+z^{N L}+z^{P L}+z^{Q L}
$$

These matrices record the $z_{i j}^{\cdot L}$ information -- the region of origin of the shipment is ignored. Along with the information, from the previous experiments, on $x^{L}$, this allows calculation of the regional technical coefficient matrices, such as $\mathrm{A}^{\mathrm{L}}$, as in (7).

Derivation of the $q_{i}^{S T}$ flows, as in (9), from the interregional data is more complicated, since both interindustry and final demand shipments must be accounted for in these $q_{i}^{S T}$. elements, while the interregional flow data, as in $z$ in (22), record only interindustry shipments; we define $\tilde{Q}(i)=\left[\begin{array}{l}\sim_{S I} \\ q_{i}\end{array}\right]$-.. here of size $(5 \times 5)$-- to be the counterpart to $Q(i)$ in (9) which contains only interindustry sales; the elements of these matrices are easily found as the i-th row sums in each of the 25 submatrices in $z$ in (22).

What is needed next is some distribution of the final demand figures, from the interregional experiments, into $(5 \times 5)$ matrices

$$
F(i)=\left[\begin{array}{c}
\mathrm{ST} \\
\mathrm{i}
\end{array}\right]
$$


recording final demand shipments of good $i$ between and within regions.

In the interregional model, the $1-t h$ element of $Y^{L}$ contains final demand uses of good 1 within region $L$ plus shipments of good i to all other regions for final demand purposes. We have chosen to allocate this $y_{1}^{L}$ to each region in the same proportion as that region's purchases of good 1 for interindustry purposes are to total interindustry sales of good $i$ by region $L$. That is,

$$
f_{i}^{S T}=\left(\begin{array}{ll}
v S T & \sim S T \\
q_{i} / \Sigma & q_{i}
\end{array}\right)\left(y_{i}^{S}\right)
$$

Then, our construction of the matrix $Q(i)$, in $(9)$, is

$$
Q(i)=\tilde{Q}(i)+F(i)
$$

The regional supply proportions are then found from these $Q(i)$, as in (11) and (12), and the model is used, as in (16) or (21). Appendix A contains a numerical example that illustrates the derivation of a multiregional input-output data set from the original interregional input-output data.

\subsection{General Structure of the Random Matrix Experiments}

Figure 1 indicates the overall structure of these experiments. There are 20 different initial $(50 \times 50) \mathrm{Z}$ matrices, from the five-region, tensector interregional case, the elements of which were randomly drawn from a uniform distribution of the integers between 0 and 100 . In conjunction with a random 50-element final demand vector, $Y$ (produced by drawing randomly from a uniform distribution of integers from 1 to 9 , then multiplying by 100), a 50-element gross output vector is found, as usual, as $X=Z i+Y$. In conjunction with the $Z^{L}$ matrices, as in (23), the regional technical coefficient matrices, $A^{L}$, as in (7), are found. 


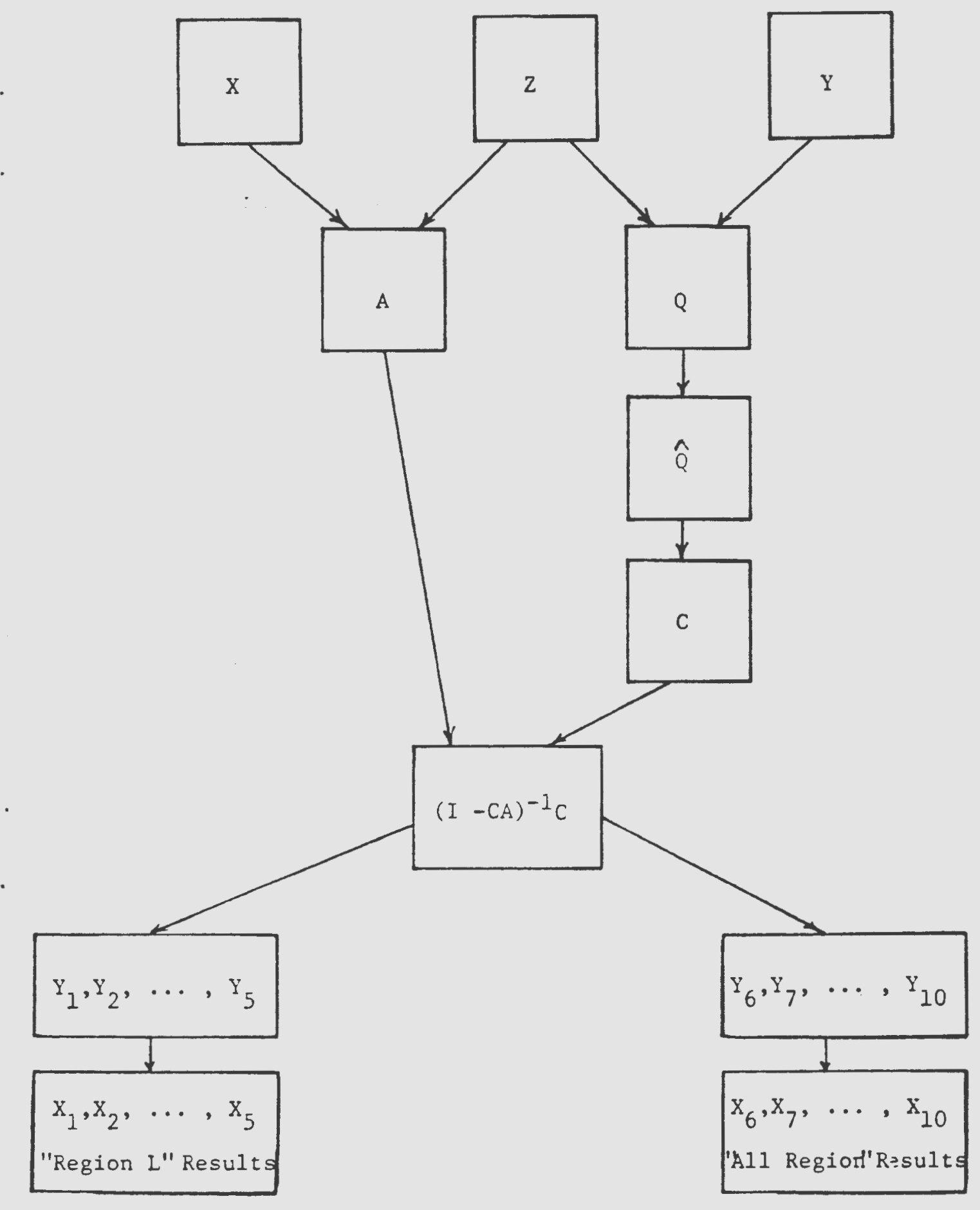

FIGURE 1: General Structure of Multiregional Spatial Aggregation Experiments $-9-$ 
In the first set of experiments $\left(Y_{1}, \ldots ., Y_{5}\right)$, only the first ten elements of any particular $Y$ were nonzero. These were the so-called "region $L^{\prime \prime}$ experiments in the interregional case. That is, these were assumed to be (new) final demands for the outputs of sectors in region L only; in the interregional input-output model the elements of $\mathrm{Y}^{\mathrm{L}}$ represent final demands within region $L$ and also exports from $L$ to all other regions for final demand uses. [of course, the region I case in the interregional model also encompasses the situation in which there is new government demand for outputs of sectors in a particular region only, for example, defense products made in California (region L).J

In the multiregional input-output model, one can also assess the impact of new government demand, in California, for outputs of the defense sectors, but with the realization that the $\hat{C}^{L L}$ and $\hat{C}^{M L}, \hat{C}^{N L}$... matrices determine the proportions of the final demand amounts that are supplied by California industries and imported into California from other regions in order to satisfy the final demand. This is a consequence of the $\hat{C} Y$ on the right-hand side of the multiregional input-output model, in (20). In these experiments, then, the random $Y_{i}(i=1, . ., 5)$ are not distributed across "receiving" regions; we interpret the nonzero elements in $Y^{I}$ to be strictly multiregional final demands, satisfied from production in $L$ or through imports to $L$. Thus the label "region $L "$ experiments has a somewhat different interpretation in the multiregional input-output experiments.

In the second set of experiments $\left(\mathrm{Y}_{6}, \ldots ., \mathrm{Y}_{10}\right)$, all 50 el ements in each $Y$ vector were nonzero. These were the "all-region" cases in the interregional input-output model, and that terminology is st1ll appropriate here. Each $Y_{i}(i=6, \ldots, 10)$, from the interregional experiments, is "reconstructed" (as 1llustrated in Appendix A) to a compatible multiregional final demand vector. 
In both region $L$ and all-region experiments, spatial aggregation then proceeds, as before, from a five-region to two-region model, with region I always spatially distinct. Thus, presentation of the results, in Sections 4 and 5, will follow exactly the same format as was used in the interregional experiments.

\subsection{Strength of Spatial Linkages}

In the interregional experiments, we introduced a measure of spatial interconnectedness, $\alpha(0 \leq \alpha \leq 1)$, by which we multiplied the elements in the off-diagonal matrices in $(22)--2^{S T}$, for $S \neq T-$ in order to alter the level of interregional dependence. As $\alpha+0$, the regions become less and less connected, economically; at $\alpha=0$, only the on-diagonal matrices -$Z^{S T}$, for $S=T$ - contain nonzero elements and the regions are not connected (economically) at all.

In the current multiregional experiments, the off-diagonal elements in each $Q(i)$-- the $q_{i}^{S I}$, for $S \neq I$-- were multiplied, in turn, by each new $\alpha$. This is equivalent to multiplying the elements in off-diagonal $2^{\text {ST }}$ matrices in $(22)$ by $a$. This produced a new set of interindustry flows, $\tilde{Q}(\dot{i})_{\alpha}$. Using the proportions from this modified $\tilde{Q}(i)$ matrix, as in (24), the original final demands were redistributed to reflect the changed intensity of spatial linkages and a modified $F(i)$ matrix was formed. Then

$$
Q(i)_{\alpha}=\tilde{Q}(i)_{\alpha}+F(i)_{\alpha}
$$

for each different $\alpha$ level. Note that in the limit, when $\alpha=0$, there will be no interregional shipments and the elements in $\mathrm{Y}^{\mathrm{I}}$ will be interpreted as final demands in region $L$ only; both $\tilde{Q}(i)_{\alpha}$ and $F(i)_{\alpha}$ (for $\alpha=0$ ) will be diagonal, and hence $Q(i)_{\alpha}$ will be diagonal also. Thus, as would be expected in the case of no economic connections between regions, all $c_{i}^{\text {II }}$ in (II) are ones, all $c_{i}^{\mathrm{ML}}$ in (12) are zeros, and hence $\hat{\mathrm{C}}$, in (18), becomes an identity 
matrix.

\subsection{Measures of Error}

In general, the same measures of error in gross outputs caused by spatial aggregation that were used for the interregional experiments were also used for the present multiregional experiments. In the interregional case, the mean absolute percentage error (MAPE) figures were calculated as follows -- where $(j)$ refers to the $j-t h$ random experiment. Let

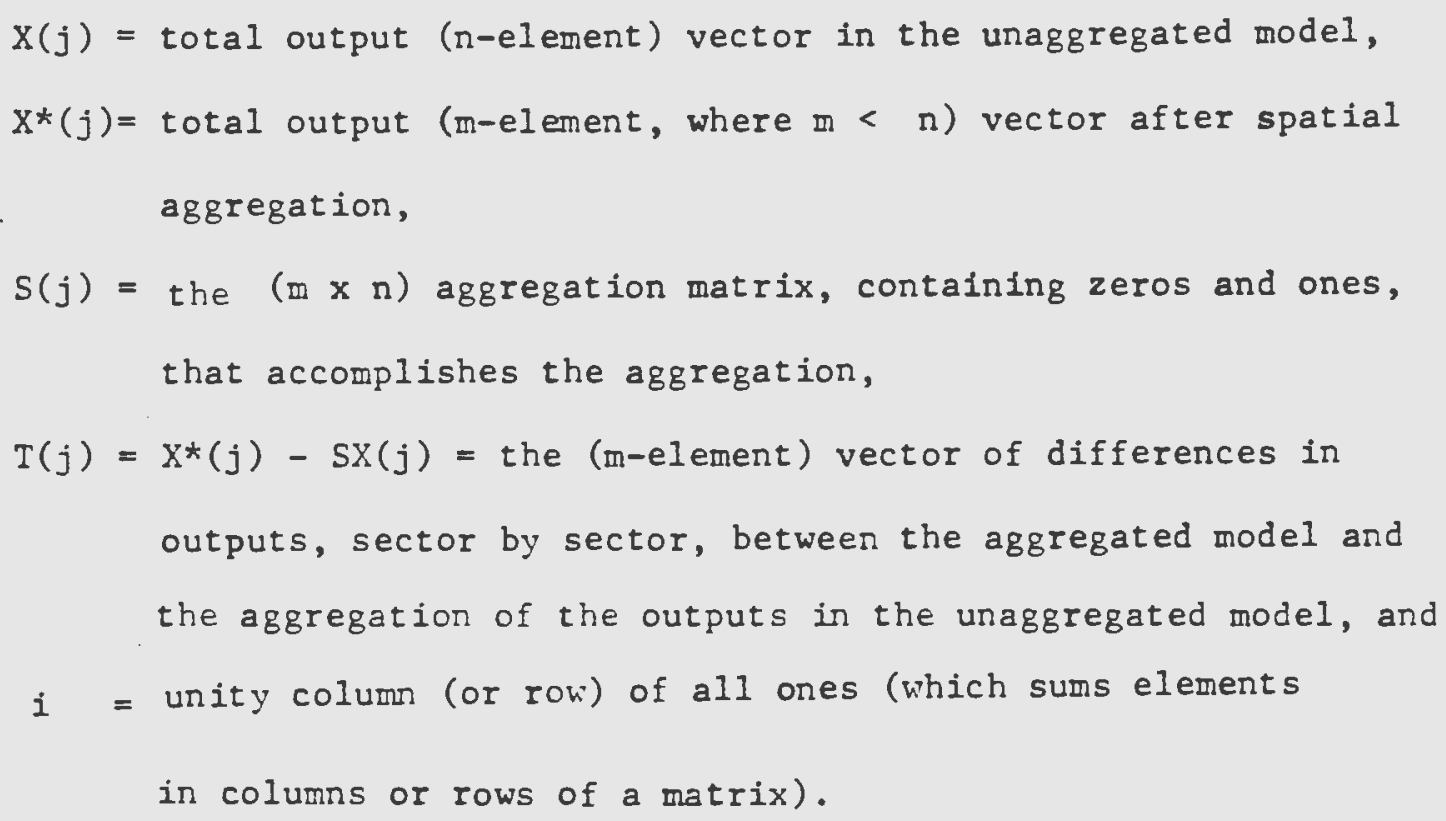

Then, in the interregional study

$$
\left.\operatorname{MAPE}_{I R}=\sum_{j=1}^{k} \frac{|i T(j)|}{i S X(j)} \times 100\right] / k
$$

where $k$ is the number of replications of the experiment. Note that the elements in $T(j)$ can be both positive and negative. For example, if $T(j)=\left[\begin{array}{l}-15 \\ +16\end{array}\right]$, $i T(j)=1$, suggesting much less error than in fact occurred. However, in the interregional experiments, virtually every element in each of the $T(j)$ vectors was negative (aggregation led to underestimation of outputs), and hence the "balancing" of positive and negative elements did not present a problem, and the MAPE's were calculated as in (27), for simplicity. 
In the multiregional experiments, this was not the case; aggregation led to underestimation of some outputs and overestimation of others. Therefore, the absolute values of each of the elements in $T(j)$ were used. Thus, for the present study

$$
\operatorname{MAPE}{ }_{M R}=\left[\begin{array}{cc}
k & i|T(j)| \\
j=1 & i S X(j)
\end{array}\right] / k
$$

The two additional measures of error that were used record aggregation bias, as defined by Theil (1957) [ see also Morimoto (1970)] -- in particular, first-order aggregation bias and total aggregation bias. The total measure, $T$, for the $j-t h$ experiment, is just

$$
T(j)=X *(j)-S X(j)
$$

which appears in the numerator in (27). First-order aggregation bias is the first term in the power-series expansion of the right-hand side of (29). Morimoto (1970, p. 121) shows first-order bias for classical (non-spatial) input-output models; here we show the straightforward extension to the multiregional case.

$$
F(j)=(\hat{C} * \hat{A} * S-S \hat{C A}) \hat{C Y}(j)
$$

where $\hat{A}$ and $\hat{A^{*}}$ are the technical coefficient matrices of the unaggregated and aggregated input-output system (for the $j$-th experiment), $\hat{C}$ and $\hat{C} *$ are the trade proportion matrices of the unaggregated and aggregated systems, and $Y(j)$ is the unaggregated final demand vector for the system (for the same experiment). ${ }^{1}$

${ }^{T}$ The use of $F(j)$, as in (30), to denote the first-order aggregation bias vector, is standard notation. It should not cause confusion with the matrices $F(i)$, whose elements, $f_{i}^{S T}$ are defined in (24). 
These (vector) measures of aggregation bias were converted to percentage figures by suming their elements (in either algebraic or absolute values), dividing by the total true (unaggregated) output, and multiplying by 100 . In particular, we define

$$
\begin{aligned}
& t(j)=\frac{1 T(j)}{1 \operatorname{Sx}(j)} \times 100 ; t=\left[\sum_{j=1}^{k} t(j)\right] / k \\
& |t(j)|=\frac{i|T(j)|}{i \operatorname{SX}(j)} \times 100 ;|t|=\left[\sum_{j=1}^{k}|t(j)| j / k\right.
\end{aligned}
$$

and

$$
\begin{aligned}
& f(j)=\frac{i F(j)}{i S X(j)} \times 100 ; f=\left[\sum_{j=1}^{k} f(j)\right] / k \\
& |f(j)|=\frac{i|F(j)|}{i S X(j)} \times 100 ;|f|=\left[\sum_{j=1}^{k}|f(j)| \mid / k\right.
\end{aligned}
$$

Thus, for the multiregional input-output experiments, $\operatorname{MAPE}_{M R}$ and $|t|$ will be the same, as shown by (28) and (32). Differences in $t$ and $|t|$ values and in $f$ and $|f|$ values reflect the presence of both positive and negative elements in $T$ and $F$, i.e., both over- and underestimation of outputs with spatial aggregation.

\section{RESULTS FROM THE RANDOM MATRIX EXPERIMENTS}

Values for the mean absolute percentage error (MAPE) in gross outputs in the multiregional model are presented in Table 1, with various levels of spatial linkage $(\alpha)$, for the region I experiments. Reading across rows, errors increase with aggregation, as might be expected. The pattern with decreasing $\alpha$ (down columns) is one of increase, then decrease, for region I output errors, and one of general increase for errors in outputs of other regions. Most importantly, however, is the observation that all errors are extremely small, never more than two percent. And the errors in ouputs in the region of particular interest in this set of experiments (region L) 
are always less than one-haif of one percent!

The structure of the region I experiments must necessarily be different in the interregional and multiregional models, and hence the MAPE results for these two models are not totally comparable. Nonetheless, it appears that for "single region" kinds of questions in either an interregional or a multiregional input-output model, a two-region framework consisting of region $L$ and the rest of the economy (e.g., California and the rest of the U.S.) is adequate in many cases.

First-order and total aggregation bias figures for these experiments are presented in Table 2. Clearly, from the differences in values for the absolute and algebraic measures, spatial aggregation in the multiregional model generates errors in both directions in total output figures. And, as expected, the $|t|$ values are the same as the MAPE's. One of the primary reasons for calculating the aggregation bias figures in the interregional case was because of a theorem on conditions under which one can be certain that first-order aggregation bias will be zero. In terms of spatial aggregation in the interregional model, the theorem says if the only final demands occur in the region or regions that remain unaggregated, then first-order bias will be zero [Miller and Blair $(1980, \mathrm{p} .6)]$. Thus, in the interregional experiments, we generated the series of region $L$ experiments, in which the only nonzero final demands were for region I sectors, in contrast with the all-region experiments, for which all elements of the final demand vector were nonzero. This allowed us to compare aggregation bias figures both with and without the first-order term, and hence provided one means of assessing the gain in accuracy that one might expect in cases where first-order bias could be eliminated. As explained in Section 3, it is impossible to generate multiregional experiments that are completely comparable to the region $I$ cases in the interregional model, and hence much of our interest in measures of first-order and total aggregation bias disappears. 
TABLE 1: MAPE in Gross Outputs -- Multiregional Model

(Random Matrices; Region L Experiments)

Level of Spatial Aggregation Outside of
Region I

Three Regions Two Regions One Region $(M, N, P+Q) \quad(M, N+P+Q) \quad(M+N+P+Q)$

$\alpha=1.00$

Output in Region L

Output Outside of Region L

All Output in the Multiregional System

$\alpha=0.75$

Output in Region $\mathrm{L}$

Output Outside of Region I

All Output in the Multi-

regional Systen

$\alpha=0.50$

Output in Region I

Output Outside of Region I

All Output in the Multi-

regional System

$a=0.10$

Output in Region L

Output Outside of Region L

All Output in the Multi-

regional system

$a=0.05$

Output in Region L

Out put Outside of Region L

All Output in the Multi-

regionl System
.127

.127

.127

.224

.251

.139

.257

.365

.175

.340

.469

.166

.321

.445

.157

.322

.463

.333

.709

1.024

.282

.598

.860

.055

.095

.129

.841

1.501

1.845

.370

.664

.041

.081

.116

.874

1.419

1.475

.267 
As reported in our previous paper, $\alpha=0.042$ for the Japanese interregional system and $\alpha$ is approximately 0.15 for Sweden; for the U.S. multiregional system [Polenske (1980)], this measure is approximately 0.0789 . There are other results on conditions under which first-order aggregation bias w1ll be zero [Morimoto (1970)], but as Table 2 suggests (using absolute value measures), for the "realistic" $\alpha=0.10$ and $\alpha=0.05$ cases, first-order bias is generally $:$ t a large proportion of the total bias produced by spatial aggregation. Hence, deriving conditions under which first-order bias would disappear in a multiregional input-output model would not appear to be a major accomplishment.

MAPE values for the all-region experiments are given in Table 3. Again, across rows, there is a general tendency for error to increase with aggregation. Also, the magnitudes of the errors for outputs in region $L$ and outside the region I are generally very similar in the least aggregated case (first column), although they become less equal as aggregation increases. The reason for the trend toward increasing error with decreasing spatial interconnectedness $(\alpha)$ escapes us, and the trend contradicts the result for the interregional model. [Table 4 repeats, for comparison, the results for the interregional model that were presented in Table 3 of Miller and Blair (1981).] Despite some differences in trends, the Table 3 results suggest that the error introduced into a multiregional input-output model through spatial aggregation is relatively small. The errors in Table 3 for $\alpha=0.10$ and $\alpha=0.05$ may be taken to be representative of real-world situations. For such cases, one can say that the error introduced by spatial aggregation in the worst case appears to be less than six percent (for the region $L$ outputs), and less than three percent, overall (for the all-region outputs). These are the same orders of magnitude as in the interregional input-output random experiments and are, in our view, not alarming. For completeness, the aggretation bias measures are shown in Table 5. 
TABLE 2: Average First-Order (f) and Total ( $t$ )

Aggregation Bias -- Multireglonal Model

(Random Matrices; Region I Experiments)

Level of Spatial Aggregation Outside of Region L

Three Regions Two Regions One Region $(M, N, P+Q)(M, N+P+Q)(M+N+P+Q)$

$\alpha=1.00$

$|f|$

$|t|$

f

$\mathrm{t}$

$\alpha=0.75$
If
$|t|$
f
$t$

$\alpha=0.50$

$|f|$
$|t|$
$f$
$t$

$\alpha=0.10$

$|f|$
$|t|$
$f$
$t$

$\alpha=0.05$
If

$t$

$t$

$$
\begin{array}{r}
0.206 \\
-0.011 \\
0.023 \\
0.000
\end{array}
$$

0.345

$-0.021$

0.020

$-0.005$

0.417

$-0.031$

0.013

$-0.003$
0.278

0.138

0.068

0.062
0.515

0.293

0.070

0.066
0.715

0.420

0.057

0.053

0.435

0.806

1.111

0.279

0.597

0.854

0.131

0.142

0.112

0.121

0.135

0.107

0.804

1.210

1.129

0.123

0.261

0.344

0.199

0.173

0.109

0.053

0.068

0.041

$|f|$
$|t|$
$f$
$t$

\begin{abstract}
0.557
$-0.040$

0.135

0.001
\end{abstract}

0.746

0.555

$-0.079$

$-0.133$

0.111

0.066

0.011

$-0.001$ 
TABIE 3: MAPE in Gross Outputs -- Multiregional Model

(Random Matrices; All-Region Experiments)

Level of Spatial Aggregation Outside

of Region I

Three Regions Two Regions One Region

$(M, N, P+Q) \quad(M, N+P+Q) \quad(M+N+P+Q)$

$\alpha=1.00$

Output in Region L

0.079

0.140

0.204

Output Outside of

Region I

0.069

0.084

0.064

All Output in the

Multiregional System

0.071

0.096

0.092

$\alpha=0.75$

Output in Region L

0.162

0.318

0.439

Output Outside of

Region L

0.175

0.324

0.449

All Output in the

Multiregional System

0.172

0.324

0.448

$\alpha=0.50$

Output in Region L

0.277

0.503

0.655

Output Outside of

Region L

0.389

0.730

0.992

Al1 Output in the

Multiregional System

0.366

0.685

0.923

$a=0.10$

Output in Region L

1.311

2.809

4.083

Output Outside of

Region L

1.505

1.920

0.994

All Output in the

Multiregional System

1.461

2.093

1.614

$\alpha=0.05$

Output in Region I

1.693

3.674

5.303

Output Outside of

Region I

1.634

$-1.960$

1.306

All Output in the Multiregional System

1.645

2.301

2.110 
TABLE 4: MAPE in Gross Outputs -- Interregional Model

(Random Matrices; All-Region Experiments)

Level of Spatial Aggregation Outside of Region L

Three Regions Two Regions One Region

$(M, N, P+Q)(M, N+P+Q)(M+N+P+Q)$

$\alpha=1.00$

Output in Region L

2.200

4.685

6.448

Output Outside of Region L

2.084

4.569

6.356

All Output in the

Interregional system

2.106

4.590

6.372

$\alpha=0.75$

Output in Region L

2.100

4.483

6.160

Output Outside of Region L

2.093

4.601

6.394

All Output in the

Interregional Syster:

2.093

4.576

6.346

$\alpha=0.50$

Output in Region $\mathrm{L}$

Output Outside of Region L

All output in the

Interregional System

1.868

3.999

5.489

2.070

4.566

6.343

2.030

4.453

6.175

$\alpha=0.10$

Output in Region L

0.718

1.545

2.125

Output Outside of Region I

All Output in the

Interregional System

1.912

4.219

5.900

1.684

3.704

5.176

$\alpha=0.05$

Output in Region $\mathrm{L}$

Output Outside of Region $\mathrm{L}$

All Output in the

Interregional System

0.403

0.868

1.195

1.879

4.135

5.798

1.599

3.509

4.920 



\section{RESULTS FROM EXPERIMENTS WITH THE U.S. MULTIREGIONAL DATA}

The existence of a set of multiregional data for the United States provided us with an opportunity to examine the effects of spatial aggregation in a real-world model. We used a nine-region, ten-sector version of the 1963 multiregional data; the nine regions are presented in Polenske (1980, Appendix A), and the ten sectors are our aggregation of the nineteen-sector data from that same source. ${ }^{2}$ Thus, the original flow matrix, $z$, is $(90 \times 90)$, and spatial aggregation proceeds, arbitrarily, by combining regions 9 and 8 , then 9,8 and 7 , etc. until, finally, we have a two-region multiregional input-output model, with region 1 and the rest of the U.S. -- a (20 x 20) Z matrix. We employ the same $(90 \times 1)$ random $Y_{i}$ vectors as were used in the Japanese interregional experiments in our earlier paper. All $y_{i}(i=1, \ldots ., 10)$ are used without any "redistribution;" this is only necessary in the random matrix cases, since those data must be made compatible with the interregional figures from which they were derived. (Again, as illustrated in Appendix A.) The MAPE figures for these experiments appear in Tables 6 and 7 .

Since the spatial interconnectedness measure for these U.S. data is 0.0789 , we can compare the errors recorded in Table 6 with those that were generated in the random region $L$ experiments for $\alpha=0.10$ (the closest $\alpha$ level used in the random cases) in Table 1. In particular, comparing the last three columns of Table 6 with the $\alpha=0.10$ results in Table 1 , we find that the random experiments underestimate the errors that occur with aggregation in the multiregional input-output model using U.S. data. For the all-region experiments, on the other hand, the $\alpha=0.10$ results in Table 3 are quite similar to those in the last three columns of Table 7 (except, perhaps, for the 4.083 in Table 3 as opposed to the 8.305 in Table 7). As in the interregional experiments, this suggests that one can study certain aspects of a

\footnotetext{
${ }^{2}$ Definitions of regions and sectors are given in Appendix $B$.
} 
real-world system with appropriately constructed random matrix experiments.

Table 8 reproduces the MAPE results for the Japanese interregional system -also nine regions of ten sectors each - that were presented in Table 11 (p. 28) of our earlier paper. Comparison of Tables 7 and 8 , as well as the $\alpha=0.10$ rows of Tables 3 and 4 , confirms the "reversal" in relative size (and seriousness) of errors in the never-aggregated region (region $L$ or region 1) and in the remainder of the economy (outside of region $L$ or region 1 ) for the all-region cases. In the interregional input-output model, aggregation creates more error in the outputs of the aggregated regions than in the outputs of the region that remains spatially distinct. Thus, for example, we find 5.900 percent vs. 2.125 percent in column 3 of Table 4 , for $\alpha=0.10$, or 3.512 percent vs. 0.136 percent in the final colum of Table 8 . By contrast, in the multiregional input-output model, it appears that the errors caused by aggregating regions are transmitted much more strongly to the unaggregated region. Again, for example, 0.994 percent vs. 4.083 percent in column 3 of Table 3 , for $\alpha=0.10$ and 0.785 percent as opposed to 8.305 percent in the final colum of Table 7. This explains the differences in the "All Output" rows for the two models at high levels of aggregation, since the figures in these rows are weighted averages of those in the preceding two rows, where the weights are the proportions of region L's (or region l's) output and all other (outside of $L$ or 1 ) regions' output to total, all-region output. In the interregional case, the larger weight is given to the larger error figure, whereas in the multiregional case it is given to the smaller figure. The information contained in the first-order and total aggregation bias figures (Tables 9 and 10 ) is much the same as 1 t was for the random experiments of the previous section. In particular, differences in $|f|$ and $f$ and in $|t|$ and $t$ values indicate both positive and negative errors in estimates of sectoral outputs as spatial aggregation occurs, and the $|f|$ figures are, again, not a large proportion of the $|t|$ values. 
TABLE 6: MAPE in Gross Outputs -- Multiregional Mode1

(U.S. Data: Region I Experiments)

Level of Spatial Aggregation Outside of Region 1

(Number of Regions)

\begin{tabular}{|c|c|c|c|c|c|c|c|}
\hline & Seven & Six & Five & Four & Three & Two & One \\
\hline Output in Region 1 & 0.001 & 0.009 & 0.013 & 0.200 & 0.211 & 0.203 & 1.525 \\
\hline $\begin{array}{c}\text { Output Outside of } \\
\text { Region } 1\end{array}$ & 0.052 & 0.296 & 0.781 & 2.064 & 2.193 & 1.902 & 3.100 \\
\hline $\begin{array}{l}\text { All Output in the } \\
\text { Multiregional system }\end{array}$ & 0.037 & 0.210 & 0.551 & 1.506 & 1.600 & 1.394 & 2.631 \\
\hline
\end{tabular}

TABLE 7: MAPE in Gross Outputs -- Multiregional Model

(U.S. Data: All-Region Experiments)

Level of Spatial Aggregation Outside of Region 1

(Number of Regions)

$\begin{array}{llllllll}\text { Output in Region 1 } & 0.474 & 0.383 & 0.396 & 1.746 & 1.579 & 3.156 & 8.305 \\ \begin{array}{c}\text { Output Outside of } \\ \text { Region 1 }\end{array} & 1.035 & 1.104 & 2.021 & 2.057 & 1.664 & 2.223 & 0.785 \\ \begin{array}{c}\text { All Output in the } \\ \quad \text { Multiregional system }\end{array} & 1.003 & 1.063 & 1.928 & 2.038 & 1.658 & 2.275 & 1.207\end{array}$


TABLE 8: MAPE in Gross Outputs - Interregional Model

(Japanese Data)

Level of Spatial Aggregation Outside of Region 1 (Number of Regions)

\begin{tabular}{|c|c|c|c|c|c|c|c|}
\hline & Seven & Six & Five & Four & Three & Two & One \\
\hline Output in Region 1 & 0.031 & 0.068 & 0.189 & 0.318 & 0.316 & 0.661 & 0.136 \\
\hline $\begin{array}{c}\text { Output Outside of } \\
\text { Region } 1\end{array}$ & 0.329 & 0.321 & 1.806 & 1.546 & 1.494 & 2.761 & 3.512 \\
\hline $\begin{array}{l}\text { All Output in the } \\
\text { Interregional system }\end{array}$ & 0.297 & 0.292 & 1.640 & 1.429 & 1.381 & 2.436 & 3.165 \\
\hline
\end{tabular}

TABLE 9: Average First-Order (f) and Total ( $t$ ) Aggregation Bias (Percent)

(U.S. Data; Region 1 Experiments)

Level of Spatial Aggregation Outside of Region 1

(Number of Regions)

$\begin{array}{cccccccc} & \text { Seven } & \text { Six } & \text { Five } & \text { Four } & \text { Three } & \text { Two } & \text { One } \\ |\mathrm{f}| & 0.020 & 0.069 & 0.118 & 0.494 & 0.114 & 0.093 & 0.987 \\ |t| & 0.037 & 0.210 & 0.551 & 1.506 & 1.600 & 1.394 & 2.631 \\ f & 0.010 & 0.004 & 0.010 & 0.035 & 0.034 & -0.002 & 0.222 \\ t & 0.028 & 0.049 & 0.080 & 0.186 & 0.278 & 0.313 & 0.873\end{array}$

TABLE 10: Average First-Order ( $f$ ) and Total ( $t$ ) Aggregation Bias (Percent)

(U.S. Data; All-Region Experiments)

Level of Spatial Aggregation Outside of Region 1

(Number of Regions)

$\begin{array}{crrrrrrr} & \text { Seven } & \text { Six } & \text { Five } & \text { Four } & \text { Three } & \text { Two } & \text { One } \\ |f| & 0.199 & 0.172 & 0.188 & 0.277 & 0.147 & 0.323 & 0.166 \\ |t| & 1.003 & 1.063 & 1.928 & 2.038 & 1.658 & 2.275 & 1.207 \\ f & -0.003 & -0.022 & -0.011 & -0.006 & 0.009 & 0.027 & -0.044 \\ t & -0.017 & -0.071 & -0.092 & -0.091 & -0.101 & 0.051 & -0.125\end{array}$




\section{CONCLUSIONS}

As in the interregional case, we would argue that spatial aggregation in multiregional input-output models produces "acceptable, not large" errors. This is based primarily on (1) the Table 3 figures for $\alpha=0.10$ and $\alpha=0.05$, and (2) the figures in Tables 6 and 7.

For questions pertaining to one or more specific regions, it appears that a multiregional input-output model in which those regions are distinct, while the rest of the economy is aggregated into the "remaining" region, is likely to be entirely adequate, at least as judged by our measures of error in sectoral outputs (Table 6). Thus, for example, because of limited computer capacity, one may wish to give up some spatial detail in order to gain sectoral detail.

Even in cases in which there are new final demands in many or all regions, questions dealing with total impacts throughout the multiregional system may be answered in a satisfactory way by using a spatially-aggregated model (Tables 3 and 7). 
Appendix A: Generating Multiregional from Interregional Data -An Illustration

For a two-region, two-sector interregional input-output example, let the randomly generated ( $4 \times 4)$ matrix 2 , as in (22), be

$(\mathrm{A}-1)$

$$
Z_{I R}=\left[\begin{array}{c:c:c}
z^{L L} & z^{L M} \\
\hdashline z^{M L} & z^{M M}
\end{array}\right]=\left[\begin{array}{rr:rr}
16 & 6 & 15 & 47 \\
339 & 89 & 43 & 42 \\
\hdashline 66 & 31 & 7 & 82 \\
90 & 92 & 91 & 78
\end{array}\right]
$$

(using subscripts $I R$ and $M R$, where necessary, for the interregional and multiregional models). Let the random $Y$ vector be

$$
(A-2) \quad Y_{I R}=\left[Y^{L}\right]=\left[\begin{array}{r}
171 \\
573 \\
32 \\
804
\end{array}\right]
$$

Thus

$$
(A-3) \quad X_{I R}=\left[\begin{array}{c}
X^{L} \\
-X^{M}
\end{array}\right]=Z_{i}+Y=\left[\begin{array}{r}
255 \\
786 \\
218 \\
1155
\end{array}\right]
$$

For the multiregional model, we $f$ ind $z^{L}$ and $z^{M}$, as in (8)

$$
(A-4) \quad z^{L}=\left[\begin{array}{rr}
82 & 37 \\
129 & 181
\end{array}\right] \text { and } z^{M}=\left[\begin{array}{rr}
22 & 129 \\
134 & 120
\end{array}\right]
$$

In conjunction with the gross outputs in $(A-3)$, we $f$ ind $A^{I}$ and $A^{M}$, as in (7)

$$
(\mathrm{A}-5) \quad \mathrm{A}^{\mathrm{L}}=\left[\begin{array}{ll}
.3216 & .0471 \\
.5059 & .2303
\end{array}\right] \text { and } \mathrm{A}^{\mathrm{M}}=\left[\begin{array}{ll}
.1009 & .1117 \\
.6147 & .1039
\end{array}\right]
$$

Finally, then, $\hat{A}$, as in $(17)$, is

$(A-6)$

$$
\hat{A}=\left[\begin{array}{cc:cc}
.3216 & .0471 & 0 & 0 \\
.5059 & .2303 & 0 & 0 \\
\hdashline 0 & 0 & .1009 & .1117 \\
0 & 0 & .6147 & .1039
\end{array}\right]
$$


In addition, from the row sums of the submatrices in $(A-1)$,

$(A-7) \quad \tilde{Q}(1)=\left[\begin{array}{ll}22 & 62 \\ 97 & 89\end{array}\right]$ and $\tilde{Q}(2)=\left[\begin{array}{cc}128 & 85 \\ 182 & 169\end{array}\right]$

Denote by $\tilde{F}(i)=\left[\tilde{p}_{i}^{n}, T_{j}\right]$ the matrices of the proportions of each of the elements in $\tilde{Q}(i)$ to the row sums of those matrices. Then

$(\mathrm{A}-8) \quad \tilde{\mathrm{P}}(1)=\left[\begin{array}{ll}.2619 & .7318 \\ .5215 & .4785\end{array}\right]$ and $\tilde{\mathrm{P}}(2)=\left[\begin{array}{ll}.6009 & .3991 \\ .5185 & .4815\end{array}\right]$

The individual elements in $Y_{I R}-(A-2)-$ are: $y_{1}^{I}=171, y_{2}^{I}=573$, $y_{1}^{M}=32$ and $y_{2}^{M}=804$. The first of these, $y_{1}^{I}$, is to be distributed according to the proportions in the top row of $\tilde{\mathrm{P}}(1)$, in $(A-8)$, that is, $y_{1}{ }_{1}=$ $(.2619)(171)=44.79$ and $y_{1}^{L M}=(.7381)(171)=126.21$. Using the proportions in the second row of $\hat{\mathrm{P}}(1)$ for $y_{1}^{M}=32$ gives $y_{1}^{M L}=(.5215)(32)=16.69$ and $y_{1}^{M}=(.4785)(32)=15.31$. The distributions of final demands for good 2 are formed similarly, from the proportions in $\tilde{\mathrm{P}}(2)$. Thus

$$
(A-9) \quad F(1)=\left[\begin{array}{ll}
44.79 & 126.21 \\
16.69 & 15.13
\end{array}\right] \text { and } F(2)=\left[\begin{array}{ll}
344.34 & 228.66 \\
416.89 & 387.11
\end{array}\right]
$$

Therefore, as in (25),

$$
(A-10) \quad Q(1)=\left[\begin{array}{ll}
66.79 & 188.21 \\
113.69 & 104.31
\end{array}\right] \text { and } Q(2)=\left[\begin{array}{ll}
472.34 & 313.66 \\
598.89 & 556.11
\end{array}\right]
$$

(Note, as pointed out in Section 2, that the row sums in $Q(i)$ equal the appropriate elements in the total gross output vector, $\mathrm{X}$ ).

From the proportions of the elements in $Q(1)$ and $Q(2)$ to the column sums in those matrices, we find, as in (13)

$(A-11) \quad C^{L I}=\left[\begin{array}{l}.3701 \\ .4409\end{array}\right], C^{L M}=\left[\begin{array}{l}.6434 \\ .3606\end{array}\right], C^{M L}=\left[\begin{array}{l}.6299 \\ .5591\end{array}\right.$, and $C^{M M}=\left[\begin{array}{l}.3566 \\ .6394\end{array}\right]$

and, therefore, $\hat{C}$ in (18) is 
$(\mathrm{A}-12)$

$\hat{\mathrm{C}}=\left[\begin{array}{cc:cc}.3701 & 0 & .6434 & 0 \\ 0 & .4409 & 0 & .3606 \\ \hdashline .6299 & 0 & .3566 & 0 \\ 0 & .5591 & 0 & .6394\end{array}\right]$

Finally, to construct the final demand vector $Y_{M R}$ from $Y_{I R}$ in $(A-2)$, we find the column sums of the $F(i)$ matrices in $(A-9)$. For example, from the first column in $F(1), y_{1}^{L L}+y_{1}^{M L}=44.79+16.69=61.48$; this is $y_{1}^{L}$ for the multregional input-output model. Similarly, from the second column in $F(1), y_{1}^{L M}+y_{1}^{M M}=126.21+15.31=141.52$, which is $y_{1}^{M}$ for the multiregional case. From column sums of $F(2)$, we find that $y_{2}^{L}=761.23$ and $y_{2}^{M}=615.77$. Therefore,

$(A-13) \quad Y_{M R}=\left[\begin{array}{l}61.48 \\ 761.23 \\ 141.52 \\ 615.77\end{array}\right]$

Column sums in the $Q(i)$ matrices -- which represent the total amounts of good $i$ that are available in each of the regions -- must be equal to the i-th row sum in the appropriate $Z$ matrix in $(A-4)$ plus the final demand sales of that good in the same region, from (A-13). For example, for good 2 in region $M$, the second column sum in $Q(2)$ in $(A-10)$ is 869.77 . From $Z^{M}$ in $(A-4)$, the sum of the elements in row 2 is 254 ; this, plus 615.77 from $Y_{M R}$ in $(A-13)$, is 869.77 .

Using the final demands in (A-13), along with $\hat{A}$ in $(A-6)$ and $\hat{C}$ in $(A-12)$, in the model structure of (21), generates precisely the original $X$ vector of gross outputs, in (A-3). That is, using $R$ to represent the reconstruction operation that transformed the vector $Y_{I R}$ into the vector $Y_{M R}$, we have

$$
\text { (A-14) } \quad X_{M R}=(I-\hat{C A})^{-1} \hat{C Y}_{M R}=X_{I R}
$$

where $Y_{M R}=R Y_{I R}$. Without this reconstruction of the "base case" $Y_{I R}$, the gross outputs generated by the multiregional input-output model that uses the base information will be different from the observed gross outputs. For 
example, using $\hat{A}$ and $\hat{C}$ as in $(A-14)$ and $Y_{I R}$ from $(A-2)$, we find (A-15) (I- $\hat{C A})^{-1} \hat{C Y}_{I R}\left[\begin{array}{r}223.66 \\ 768.05 \\ 242.52 \\ 1169.50\end{array}\right]$

which is not the same as the gross output vector in $(A-3)$. 
Appendix B: Regions and Sectors in the U.S. Multiregional Data

Regions

\begin{tabular}{|c|c|c|}
\hline Number & Name & Inclusive States \\
\hline 1 & New England & $\begin{array}{c}\text { Connecticut, Maire, Massachusetts, New } \\
\text { Hampshire, Rhode Island, Vermont }\end{array}$ \\
\hline 2 & $\begin{array}{l}\text { Middle Atlan- } \\
\text { tic }\end{array}$ & New Jersey, New York, Pennsylvania \\
\hline 3 & $\begin{array}{l}\text { East North } \\
\text { Central }\end{array}$ & Illinois, Indiana, Michigan, Ohio, Wisconsin \\
\hline 4 & $\begin{array}{l}\text { West North } \\
\text { Central }\end{array}$ & $\begin{array}{l}\text { Iowa, Kansas, Minnesota, Missouri, Nebraska, } \\
\text { North Dakota, South Dakota }\end{array}$ \\
\hline 5 & $\begin{array}{l}\text { South Atlan- } \\
\text { tic }\end{array}$ & $\begin{array}{l}\text { Delaware, District of Columbia, Florida, } \\
\text { Georgia, Maryland, North Carolina } \\
\text { South Carolina, Virginia, West Virginia }\end{array}$ \\
\hline 6 & $\begin{array}{l}\text { East South } \\
\text { Central }\end{array}$ & Alabama, Kentucky, Mississippi, Tennessee \\
\hline 7 & $\begin{array}{l}\text { West South } \\
\text { Central }\end{array}$ & Arkansas, Louisiana, Oklahoma, Texas \\
\hline 8 & Mountain & $\begin{array}{l}\text { Arizona, Colorado, Idaho, Montana, Nevada, } \\
\text { New Mexico, Utah, Wyoming }\end{array}$ \\
\hline 9 & Pacific & $\begin{array}{l}\text { California, Oregon, Washington, Alaska, } \\
\text { Hawaii }\end{array}$ \\
\hline
\end{tabular}

\section{Sectors}

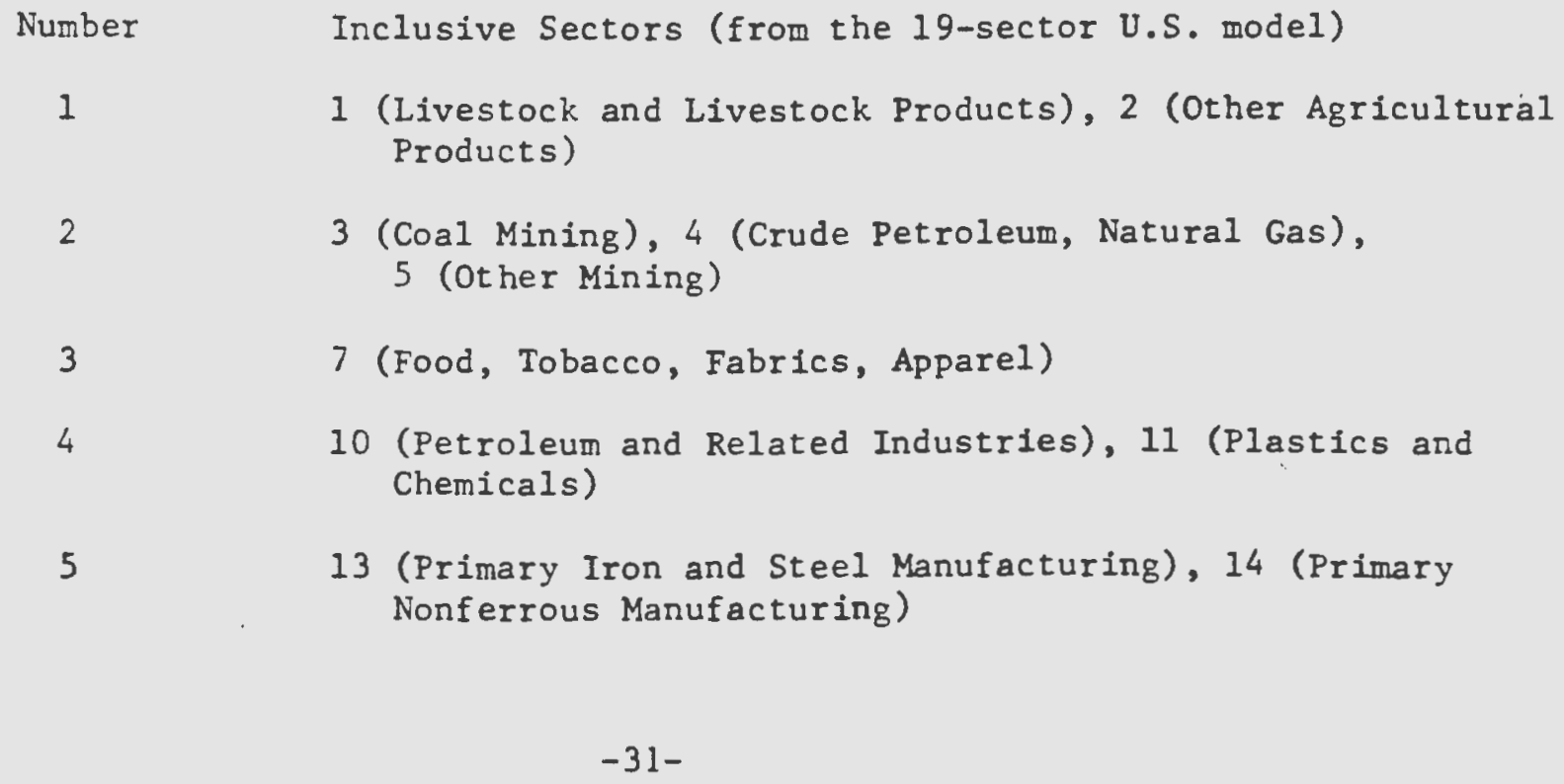


8 (Transportation Equipment and Ordnance), 15 (Machinery and Equipment)

12 (Glass, Stone and Clay Products)

8

6 (Construction)

9

17 (Transportation and Warehousing)

10

9 (Lumber and Paper), 16 (Services), 18 (Gas, Water and Sanitary Services), 19 (Electric Utilities) 
Miller, Ronald E. and Peter Blair. "Spatial Aggregation in Interregional InputOutput-Models." Working Papers in Regional Science and Transportation, No. 35. University of Pennsylvania, Philadelphia, Pennsylvania, October, 1980. (A modified version of this paper is forthcoming in Papers, Regional Science Association, Vol. 47, 1981.)

Morimoto, Y. "On Aggregation Problems in Input-Output Analysis," Review of Economic Studies, Vol. 37 (1970), pp. 119-126.

Polenske, Karen R. The U.S. Multiregional Input-Output Accounts and Model. Lexington, MA: Lexington Books, 1980.

Theil, Henri. "Linear Aggregation in Input-Output Analysis," Econometrica, Vo1. 25 (1957), PP. 111-122. 